delay between the causal incident and the appearances of radiographic abnormalities. The radiographic abnormalities may be classified in three types: type A, juxta articular lesions leading to osteoarthritis; type $\mathrm{B}$, head, neck and shaft lesions; type $\mathrm{C}$, lesions looking like bone island. Early detection of the bone necrosis is important to monitor compressed air workers. Detection depends on good quality radiographs (modifications of the trabeculae in a susceptible area). Bone areas of bone damage may go completely unrecognised on a radiograph. Lesions, early or doubtful, can be confirmed by MRI or bone scintigraphy but only $18 \%$ of the positive bone scintigraphy will develop radiographic abnormalities.

Survey of caisson workers is necessary because they can develop lesions in previously normal areas in the absence of further exposure to hyperbaric pressures. It is not certain that a repair process always takes place and it is impossible to detect juxta articular lesions that do not progress to structural failure of the joint surface and those that are going to progress to a secondary degenative joint disease. So, there may be a long delay in making a diagnosis in an individual susceptible worker resulting in continous of the same type of work and thus exposure to the risk of further bone damage.

There is no good preventive treatment: prevention by the use of official decompression tables seems to be inadequate because decompression sickness tends to be underreported related to the delay of diagnosis and it has been found that the tables do not decrease the number of dysbaric osteonecrosis. Oxygen decompression seems to be one of the only viable method for the safely decompressing tunnel workers.

\section{SP0085 MUSIC AND RHEUMATISM}

W Grassi, R De Angelis, A Farina, C Cervini. Department of Rheumatology, University of Ancona, Jesi, Italy

10.1136/annrheumdis-2001.32

The relationship between music and rheumatism is strong, complex, and interesting. It can be classified into five general categories:

- Occupation-specific musculoskeletal diseases in musicians due to repetitive strain injury;

- Joint hypermobility-related benefits among musicians;

- Joint hypermobility-related disadvantages among musicians;

- Rheumatological pathography of famous composers (e.g., Beethoven, Schumann, Paganini);

- Joint music (acoustical analysis of joint sounds by digital phonoarthrometry);

- Music therapy for pain and anxiety management.

Musculoskeletal symptoms are highly prevalent among musicians at all levels of performance. ${ }^{1}$

This is not surprising in the context of activities involving repetitious movement and/or postural constraint. Increased tension in the affected muscles could play an important causative role.

Playing-related musculoskeletal disorders in musicians (PRMD) are chronic and disabling conditions that may lead to the loss of a career and incomes because of the weakness of the executant muscle actions and loss of agility, speed and accuracy. PRMD are also under-recognised and under-researched causes of pain and disability especially in music students. Data from previous surveys indicate that the prevalence of PMRD in adult classical musicians is comparable to the prevalence of work- related musculoskeletal disorders reported for other occupational groups.

Clinical findings of PMRD are deeply related to size, weight, playing position of the instrument, playing time, posture, and technique.

Soft tissue rheumatism and predisposition to injury are common associations of joint hypermobility among musicians. ${ }^{2}$ However, joint hyperlaxity may confer advantages in playing some instruments such as flute, piano, and violin. ${ }^{3}$ The outstanding virtuosity of the violinist Niccolò Paganini has been related to the remarkable laxity of his joints.

Hand pain is a very common complaint among classical musicians. Clinical syndromes include: piano and guitar cramp, traumatic synovitis of the wrist joint, dystonia, carpal tunnel syndrome, acro-osteolysis, ulnar neuropathy and premature closure of the growth of the fingers in adolescents.

The onset of clinical features is generally associated with an increase in playing or practice load.

Hand difficulties are more frequent among pianists. They include: pain, weakness, tightening, cramping, curling and drooping, and loss of control.

The diagnosis of PRMD can be difficult when symptoms are mild and only occur on playing.

The management of PMRD is often difficult. Early and regular prevention, rest, splinting, correction of technical problems by skilled teachers of the instrument, and ergonomic redesign of some instruments can be of critical importance. Non steroidal anti-inflammatory drugs and local steroid injection can provide good symptomatic relief. Occupational therapy can be very useful. ${ }^{4}$ Trills, arpeggios or octaves should be avoided or reduced in pianists with hand complaints. Some composers such as Tchaikowsky (Piano Concerto N. 1 in B-flat Minor) or Beethoven (Piano Concerto N. 3 in C Minor; Sonata in E Major, opus 109; Sonata in C Major, opus 2 N. 3) seem to be at higher risk of worsening hand pain and inability. ${ }^{5}$

Music therapy as a treatment for chronic pain disorders and for depression has been proposed.

\section{REFERENCES}

1 Zaza C. Playing-related musculoskeletal disorders in musicians: a systematic review of incidence and prevalence. CMAJ 1998;158:1019-25

2 Fry $\mathrm{HJH}$. Overuse syndrome in musicians: prevention and management. Lancet 1986;ii:728-31

3 Grahame R. Joint hypermobility and the performing musician. N Engl J Med. 1993:329:1120-1

4 Hochberg FH, Leffert RD, Heller MD, Merriman L. Hand difficulties among musicians. JAMA 1983:249:1869-72

5 Cervini C, Gasparini M, Grassi W. La "sindrome da bandoneòn". Proceedings of the XXVII Congresso Nazionale della Società Italiana di Reumatologia. Montecatini Terme, 30 October-2 November 1986. Abstract no. 172

\section{SP0086 OCCUPATIONAL PHYSICAL ACTIVITIES AND KNEE OSTEOARTHRITIS}

D Coggon. MRC Environmental Epidemiology Unit, Southampton General Hospital, Southampton, UK

\subsection{6/annrheumdis-2001.33}

Assessing the contribution of occupational activities to knee osteoarthritis presents several methodological difficulties. Nevertheless, a strong body of evidence has accumulated that the disease is more common in people who have carried out heavy physical work, and particularly in those whose work has entailed prolonged or repeated kneeling or squatting. The precise 
definition of occupational kneeling and squatting has varied between studies, but relative risks have often been two or higher.

It seems likely that the hazard arises from unusual mechanical stresses on the joint, and it is notable that the same occupational activities have also been linked with degenerative tears of the menisci. In addition, some occupations such as professional footballers carry a risk of acute meniscal injury which may later lead to osteoarthritis.

The challenge now is to translate these epidemiological observations into effective policies for the prevention of disease. In most developed countries, the requirement for heavy physical work has declined over recent decades as a consequence of increasing mechanisation. However, there are still many jobs that involve kneeling or squatting. Often, as for example in the construction industry, the work work environment is difficult to control and the hazardous tasks cannot easily be eliminated or substituted. Knee pads may be used to reduce discomfort and protect against pre-patellar bursitis, but as yet we have no evidence that they reduce the risk of osteoarthritis.

Another approach might be to identify individuals who are at unusually high risk from kneeling because of the concomitant presence of other risk factors. For example, in a recent case-control study, the interaction of occupational kneeling with obesity was approximately multiplicative. It follows that the absolute reduction in risk from avoidance of obesity would be greater in workers who have to kneel in their jobs. Where the combination of occupational kneeling with other risk factors leads to exceptionally high risk, there may even be a case for selectively excluding such individuals from certain jobs.

\section{Physiology of endothelial cell - Friday 15 June, 16.00-17.30/South Hall}

\section{SP0087 TISSUE ENGINEERING AND STEM CELL BIOLOGY: DEVELOPMENT OF BONES, JOINTS AND SYNOVIUM}

N Zvaifler. UCSD, Rheumatology, La Jolla, CA, USA

\subsection{6/annrheumdis-2001.34}

Skeletal morphogenesis begins in structures called limb buds 41 / 2 weeks after gestation. The limb buds, comprised of mesodermal cells, form at discreet locations beneath a layer of ectoderm. The initial stimuli for limb development are still vague, but FGF-8 and possibly retinoic acid are important. By week 5 the limb primordium begins to bulge from the body wall. Despite the bud's simple structure it contains enough information to guide its development, for if transplanted to another site or cultured in vitro it will become a limb. At the apex of the bud is a thickened ectoderm (apical ectodermal ridge) and experiments have shown that continuous interactions between the AER and underlying mesoderm promote the initiation phase, outgrowth phase, and subsequent skeletal morphogenesis. The limb develops along three axis's; proximodistal (base of limb to digits); anteroposterior (digits 1 to 5); dorsoventral (top of hand/foot to palm/sole). Each is under the influence of "morphogens" made in discreet signalling centres.

\section{Abstract SP0087 Table 1}

\begin{tabular}{lll}
\hline Axis & Signalling Centre & Molecular signals \\
\hline Proximodistal & Apical ectodermal ridge (AER) & FGF-2, 4, 8 \\
Anteroposterior & Zone of polarising activity (ZPA) & Sonic hedgehog \\
Dorsoventral & Dorsal ectoderm & Wnt-7a (dorsal) \\
& Ventral ectoderm & En-1 (ventral) \\
\hline
\end{tabular}

Skeletal elements of the limbs first appear in a core of mesenchymal cells, then in cartilage (weeks $51 / 2$ to $61 / 2$ ), and finally in bone ( 6 to 8 weeks). The condensed cells that form the precartilage express bone morphogenetic proteins (BMP-2 and -4). As development continues they are restricted to cells that will become periosteum or perichondrium.

In contrast, BMP-6 is only expressed in maturing cartilage within limbs. Another signalling molecule, Indian hedgehog, found in the same location is thought to induce the BMP-6 expression.

Joint formation is a late feature in limb differentiation. Around week 6, between condensations in the precartilage, there develop transverse bands of tightly packed, flat mesenchymal cells, called interzones. At this time, growth/differentiation factor-5, a member of the BMP family, is expressed in the condensed cells. The interzone consists of two regions of high cell density surrounding a region of low cell density. The former will become articular cartilage, while the latter disappears through apoptosis leaving behind a cavity. At the periphery of each interzone are mesenchymal cells and blood vessels. These arise from the primordial perichondrium and will become the future synovium and joint capsule.

The synovium consists of an incomplete lining (the intima) sitting on a vascularized matrix. After birth two cell types can be identified. One (the type A synoviocyte) arises in the bone marrow, passes into the circulation and enters the joint as a monocyte, but during its progression into the intima acquires attributes of a tissue macrophage (including the CD68 phenotype). The other lining cell is a specialised fibroblast, similar to bone marrow stromal cells that supports hematopoeisis (type B fibroblast-like synoviocyte). However, these only acquire VCAM-1 and the typical FLS phenotype after birth.

The shafts of long bones (diaphysis) are completely ossified at birth, but the ends of the bones (epiphysis) are still cartilagenous. Only later, when secondary centres of ossification develop does this segment gradually become bone. Even then, however, a layer of cartilage persists between the epiphysis and the growing end of the diaphysis. In man chondrocyte proliferation continues in this growth plate for about twenty years and not until epiphyseal fusion does bone growth cease.

\section{SP0089 CELL-BASED TECHNOLOGIES IN JOINT REPAIR}

F Luyten. Rheumatology, University Hospitals KU Leuven, Leuven, Belgium

10.1136/annrheumdis-2001.35

Tissue engineering is a field of Biomedicine that is rapidly growing, and is critically driven by scientific advances in the areas of developmental and cell biology, material sciences and biochemistry/polymer chemistry. Regeneration of skeletal tissues, including joint surface repair, skin repair, and treatment of CNS disorders are among the most promising areas, and are closest to 